\title{
Metabolomic Profile of Aggressive Meningiomas by Using High- Resolution Magic Angle Spinning Nuclear Magnetic Resonance
}

\author{
Laura Bender, ${ }^{* \dagger \odot}$ François Somme, Elisa Ruhland, ${ }^{\ddagger}$, A. Ercüment Cicek, ${ }^{\dagger, \perp}$ Caroline Bund, ${ }^{\ddagger}, \AA$ \\ and Izzie Jacques Namer ${ }^{\ddagger}$, ,\# \\ ${ }^{\dagger}$ Oncology Department, ${ }^{\ddagger}$ Biophysics and Nuclear Medicine Department, ${ }^{\S}$ MNMS-Platform, University Hospitals of Strasbourg, \\ Hôpital de Hautepierre, 1 Avenue Molière, Strasbourg 67200, Alsace, France \\ "Computational Biology Department, School of Computer Science, Carnegie Mellon University, Pittsburgh 15213, Pennsylvania, \\ United States \\ ${ }^{\perp}$ Computer Engineering Department, Bilkent University, Ankara 06800, Turkey \\ \#ICube, Université de Strasbourg/CNRS, UMR 7357, Strasbourg 67081, Alsace, France
}

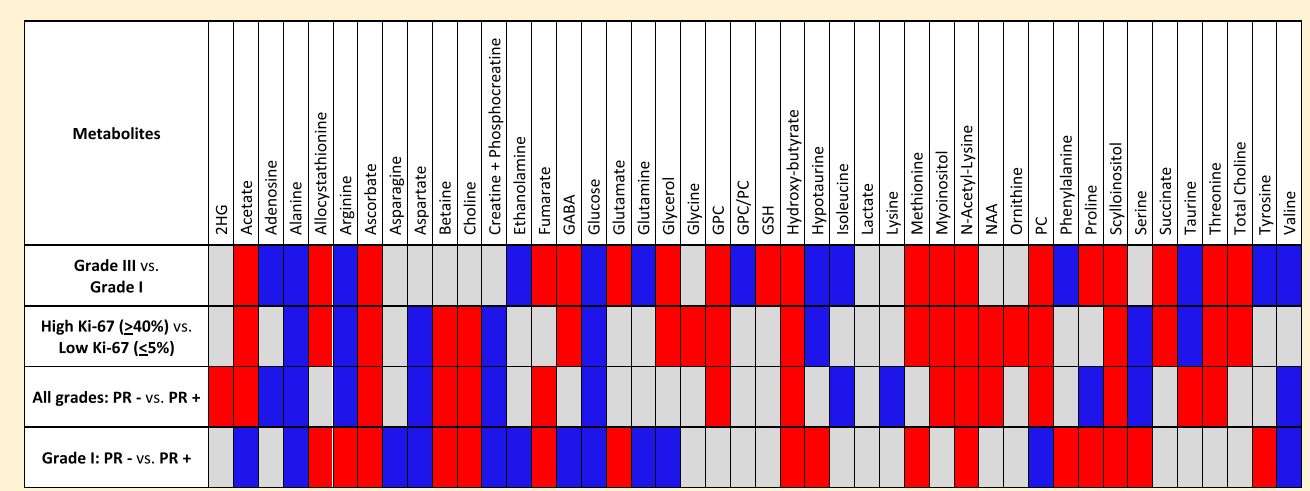

ABSTRACT: Meningiomas are in most cases benign brain tumors. The WHO 2016 classification defines three grades of meningiomas. This classification had a prognosis value because grade III meningiomas have a worse prognosis value compared to grades I and II meningiomas. However, some benign or atypical meningiomas can have a clinical aggressive behavior. There are currently no reliable markers which allow distinguishing between the meningiomas with a good prognosis and those which may recur. High-resolution magic angle spinning (HRMAS) spectrometry is a noninvasive method able to determine the metabolite profile of a tissue sample. We retrospectively analyzed 62 meningioma samples by using HRMAS spectrometry (43 metabolites). We described a metabolic profile defined by a high concentration for acetate, threonine, $N$-acetyl-lysine, hydroxybutyrate, myoinositol, ascorbate, scylloinositol, and total choline and a low concentration for aspartate, glucose, isoleucine, valine, adenosine, arginine, and alanine. This metabolomic signature was associated with poor prognosis histological markers $[\mathrm{Ki}-67 \geq 40 \%$, high histological grade and negative progesterone receptor (PR) expression]. We also described a similar metabolomic spectrum between grade III and grade I meningiomas. Moreover, all grade I meningiomas with a low Ki-67 expression and a positive PR expression did not have the same metabolomic profile. Metabolomic analysis could be used to determine an aggressive meningioma in order to discuss a personalized treatment. Further studies are needed to confirm these results and to correlate this metabolic profile with survival data.

KEYWORDS: ex vivo spectrometry, HRMAS NMR, meningioma, metabolic signature

\section{INTRODUCTION}

Meningiomas are the most common adult primary central nervous system tumors. The Central Brain Tumor Registry of the United States (CBTRUS) reported 129841 new cases between 2008 and 2012. In the United States, meningiomas represent $36.4 \%$ of all cases of primary central nervous system tumors. $^{1,2}$ Meningiomas derive from arachnoid cap cells located in the arachnoid villi. These tumors arise in the majority of cases from brain meninges, but $10 \%$ derive from spinal cord meninges. ${ }^{3}$ Immunohistochemical analysis reveals an expression of vimentin, protein S100, epithelial membrane antigen, and progesterone receptors (PRs). ${ }^{4,5}$ Ragel and Jensen described aberrant signaling pathways (mammalian target of rapamycin, phosphoinositide 3-kinase, and mitogen-activated protein kinase) implicated in meningioma tumorigenesis. ${ }^{6}$ According to the World Health Organization (WHO) 2016 classification, meningiomas are divided into three grades: grade I or benign meningiomas, grade II or atypical meningiomas, and grade III or malignant meningiomas. The WHO 2016

Received: August 3, 2019

Published: November 4, 2019 
classification did not undergo revisions concerning the classification and the grading of meningiomas compared to the WHO 2007 classification. The only change was that brain invasion was added to the criteria, which suffices to diagnose grade II meningiomas. Grade I meningiomas (nine subtypes) represented the most common variant. These tumors had a good prognosis with a 10 year progression-free survival (PFS) rate from 75 to $95 \%$ and a 10 year overall survival (OS) rate from 80 to $90 \%$. $^{7}$ Grade II meningiomas (atypical, clear-cell, and chordoid) had a poor prognosis with a 10 year PFS rate from 23 to $78 \%$ and a 10 year OS rate from 50 to $79 \%$. $^{7}$ Grade III meningiomas (anaplastic, papillary, and rhabdoï) are a rare variant of meningiomas. These tumors represent $1.2 \%$ of all meningiomas. Grade III meningiomas are defined by 20 or more mitoses per 10 high-power fields and/or pathological examinations, which look like pseudocarcinomas, pseudomelanomas, or high-grade pseudosarcomas. ${ }^{8}$ These tumors had a worse prognosis with a 10 year PFS rate of $0 \%$ and a 10 year OS rate from 14 to $34 \% .^{7}$ Patients with grade III meningiomas had a worst prognosis compared to patients with grade I and II meningiomas because of a higher risk of recurrence and their capacity to develop brain and distant metastases. However, some grade I and grade II meningiomas may present a clinical aggressive behavior. Cellular proliferation is based on protein synthesis, and amino acids (AAs) are the natural building blocks of protein. Monitoring AA expression by using highresolution magic angle spinning (HRMAS) spectrometry is interesting to understand the physiological and pathological processes of cellular metabolism. ' HRMAS nuclear magnetic resonance (NMR) spectrometry is a nondestructive method that is used to determine the metabolomic profile of a tumor sample. A tissue sample preparation is essential. ${ }^{10}$ This technique has already been evaluated in several fields, notably to explore acute rejection after tissue transplantation or to improve the diagnosis and staging of tumor in oncology. ${ }^{11-13}$ In the actual literature, two studies evaluated the metabolite profile of meningiomas by using HRMAS. Monleón et al. analyzed 10 metabolites according to HRMAS spectrometry in 30 meningioma samples. ${ }^{14}$ Pfisterer et al. analyzed 68 meningioma samples (46 grade I, 14 grade II, and 8 grade III) by using proton magnetic resonance spectroscopy (8 metabolites). ${ }^{15}$ Ex vivo spectroscopy analysis could permit to distinguish between meningioma with a good prognosis and those which tend to recur. Actually, there is a lack of data considering the spectroscopy analysis of meningiomas by using HRMAS NMR. The aim of this study was to correlate a metabolic profile with aggressive histological features for the meningiomas.

\section{MATERIALS AND METHODS}

\section{HRMAS NMR Analysis}

Sample Preparation. Tissue specimens were collected with minimum ischemic delays after resection (average time 2 $\pm 1 \mathrm{~min}$ ) and snap-frozen in liquid nitrogen before being stored at $-80{ }^{\circ} \mathrm{C}$. All tissue samples used in this study had a viable tumor/necrosis ratio, which was quantitatively and qualitatively adequate to perform satisfactory NMR HRMAS analysis. In order to wait for this goal, after NMR HRMAS analysis, the inserts were cut, and for half the content of each sample, the percentage of tumor cells in the total sample of cells with regard to the total surface was calculated using frozen hematoxylin and eosin-stained sections. Only samples containing more than $80 \%$ of tumor cells were kept for the study. Each brain biopsy sample was prepared at $-20{ }^{\circ} \mathrm{C}$ by introducing a $15-18 \mathrm{mg}$ biopsy into a disposable $30 \mu \mathrm{L} \mathrm{KelF}$ insert. To provide a lock frequency for the NMR spectrometer, $10 \mu \mathrm{L}$ of $\mathrm{D}_{2} \mathrm{O}$ was also added to the insert.

HRMAS NMR Data Acquisition. All HRMAS NMR spectra were acquired on a Bruker (Karlsruhe, Germany) AVANCE III 500 spectrometer operating at a proton frequency of $500.13 \mathrm{MHz}$ and equipped with a $4 \mathrm{~mm}$ tripleresonance gradient HRMAS probe $\left({ }^{1} \mathrm{H},{ }^{13} \mathrm{C}\right.$, and $\left.{ }^{31} \mathrm{P}\right)$. The temperature was maintained at $4{ }^{\circ} \mathrm{C}$ throughout the acquisition time in order to reduce the effects of tissue degradation during the spectrum acquisition. A one-dimensional (1D) proton spectrum using a Carr-PurcellMeiboom-Gill (CPMG) pulse sequence was acquired with a $285 \mu$ s interpulse delay and a $10 \mathrm{~min}$ acquisition time for each tissue sample. The number of loops was set at 328 , giving the CPMG pulse train a total length of $93 \mathrm{~ms}$. The chemical shift was calibrated to the peak of the methyl proton of L-lactate at $1.33 \mathrm{ppm}$. To confirm resonance assignments in a few representative samples, two-dimensional heteronuclear experiments $\left({ }^{1} \mathrm{H}-{ }^{13} \mathrm{C}\right)$ were also performed immediately after ending the $1 \mathrm{D}$ spectra acquisition.

HRMAS NMR Data Processing. Metabolite assignment and quantification were done with Chenomx software (Edmonton, $\mathrm{AB}$, Canada) using a database of NMR spectra of 76 metabolites acquired in our laboratory under the same CPMG pulse sequence as the tissue samples. ${ }^{16}$ We could detect and quantify 43 metabolites in meningioma samples: acetate, adenosine, alanine, allocystathione, arginine, ascorbate, asparagine, aspartate, betaine, choline, creatine, ethanolamine, fumarate, $\gamma$-aminobutyric acid (GABA), glycine, glucose, glutamate, glutamine, glutathione (GSH), glycerol, glycerophosphocholine (GPC), hydroxybutyrate (HB), 2-hydroxyglutarate $(2 \mathrm{HG})$, hypotaurine, isoleucine, lactate, lysine, methionine, myoinositol, $\mathrm{N}$-acetyl-aspartate (NAA), $\mathrm{N}$-acetyllysine (NA-lysine), ornithine, phenylalanine, phosphocholine (PC), phosphocreatine, proline, serine, scylloinositol, succinate, taurine, threonine, tyrosine, and valine. The results are expressed in $\mathrm{nmol} \cdot \mathrm{mg}^{-1}$ of tissue. We also used total choline (choline + GPC + PC) and total creatine (creatine + phosphocreatine) as additional parameters in a network analysis.

\section{Network Analysis}

The algorithm to determine the expected metabolite level alterations (ADEMA) network analyses using mutual information were applied to the metabolite quantification value. ${ }^{17}$ ADEMA include information on the metabolic pathway in a unidirectional or bidirectional manner. The network was constructed using the Kyoto Encyclopedia of Genes and Genomes $^{18,19}$ and Salway's work. ${ }^{13}$ Using the metabolic network topology, the ADEMA algorithm evaluates the change in groups of metabolites between concentration data from two experimental groups instead of analyzing metabolite concentrations one by one. Based on mutual information, the algorithm determines whether some metabolites are biomarkers when considered together, and it can predict the direction of the expected change per metabolite depending on the metabolic network topology considered. Various groups of metabolites related to the metabolic pathways involved were compared: 


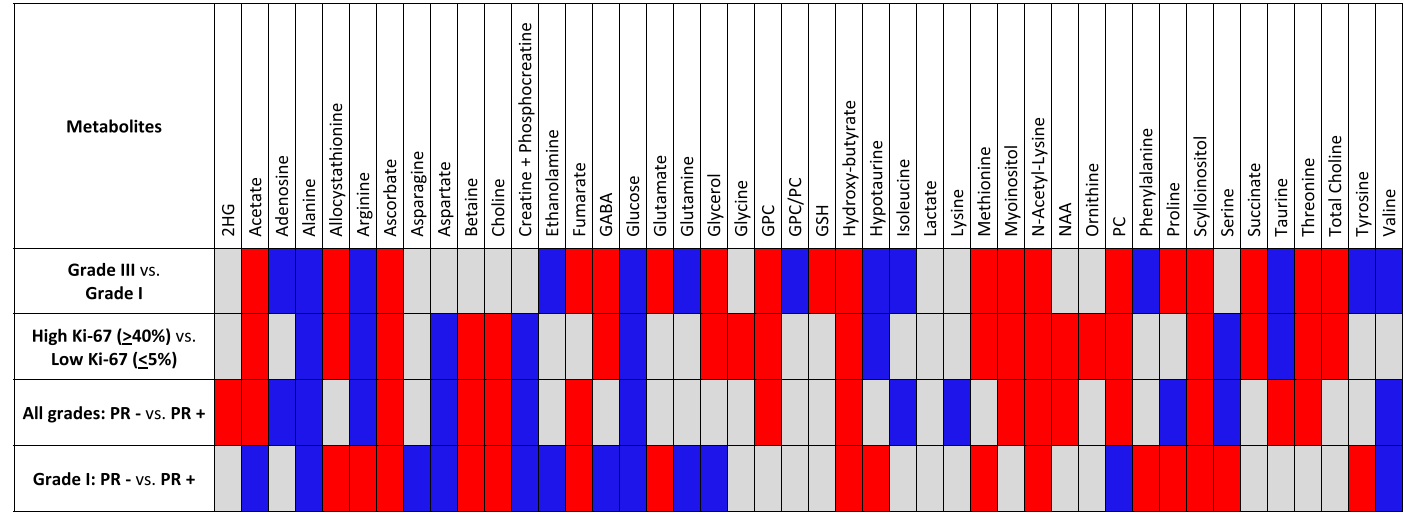

Figure 1. Summary of ADEMA network analysis conducted for specified group comparison based on the metabolite concentration obtained by HRMAS NMR spectroscopy. Red and blue boxes indicate higher and lower metabolite concentrations, respectively, between groups and gray boxes indicate no significant difference.

- Taurine, hypotaurine, aspartate, methionine, allocystathione, and serine

- Aspartate, asparagine, acetate, threonine, and NAA

- Aspartate, lysine, and NA-lysine

- Acetate, threonine, allocystathione, and methionine

- Glucose, acetate, and HB

- Aspartate, threonine, and isoleucine

- Glucose, glycine, and serine

- Glucose, glycerol, phenylalanine, and tyrosine

- Glucose, valine, and isoleucine

- Glucose and lactate

- Valine, lactate, and alanine

- Glucose, myoinositol, ascorbate, GSH, glycine, and glutamate

- Myoinositol and scylloinositol

- Glutamate, GABA, and proline

- Aspartate, adenosine, succinate, fumarate, and $2 \mathrm{HG}$

- Glutamate, glutamine, glycine, and $2 \mathrm{HG}$

- Glutamate, arginine, glycine, creatine, and ornithine

- Aspartate, arginine, and ornithine

- Ethanolamine, choline, GPC, PC, and total choline

- Choline, betaine, and glycine

Histological Analysis

We studied 62 surgical samples. Hematoxylin and eosin staining was done for histological typing. Tumors were graded according to the WHO 2016 (world health organization) classification. Immunohistochemical staining was done to determine Ki-67 (clone SP6) and PR (clone 16).

Written patient's consent has been obtained. The Ethics Committee of Strasbourg approved the study (CARMeN Project, Ethics Committee no. 2003-100, 09.12.2003).

\section{Statistical Analysis}

The receiver operating characteristic curve was used to define the optimal threshold of $\mathrm{Ki}-67$ to distinguish between grade I and grade II/III meningiomas in our cohort (Youden index). Thus, the optimal cutoff obtained was $12.5 \%$ (AUC 88.3, sensitivity $87.5 \%$, and specificity $97.8 \%$ ).

\section{RESULTS}

\section{Patient Population}

We retrospectively included 62 meningiomas from 50 patients treated by surgery in the Department of Neurosurgical at University Hospitals of Strasbourg between October 2002 and
September 2010. There were 45 grade I, 8 grade II, and 6 grade III meningiomas. The histological grade was statistically associated with OS $(p<0.0001)$.

Forty-three meningiomas had a Ki-67 rate under 5\%, while eight tumors had a Ki-67 rate over $40 \%$. For three patients, the Ki-67 expression was not available. A high Ki-67 rate (>40\%) was statistically associated with a worser OS $(p=0.0017)$. The PR expression was obtained for 54 meningiomas, among which 33 were positive and 21 were negative. A positive PR expression was statistically associated with a longer OS $(p<$ 0.0001 ). Among the 62 tumor samples, 58 (93\%) were primary tumors and four were meningioma-relapsed.

\section{Metabolic Spectrum According to Histological Grade}

Meningiomas with a high histological grade (II and III) according to the WHO 2007 classification were associated with an elevated concentration for acetate, threonine, NA-lysine, glycine, myoinositol, ascorbate, scylloionitol, HB, succinate, choline, GPC, PC, total choline, and glycerol and a low concentration for taurine, aspartate, serine, glucose, isoleucine, valine, alanine, adenosine, glutamine, arginine, ethalonamine, and betaine.

\section{Metabolic Spectrum According to Ki-67 Expression}

A high Ki-67 rate was statistically associated with an elevated concentration of allocystathionine, methionine, acetate, threonine, NAA, NA-lysine, HB, glycine, myoinositol, ascorbate, scylloinositol, GABA, succinate, ornithine, and choline and a low concentration of taurine, hypotaurine, aspartate, serine, glucose, alanine, adenosine, arginine, creatine, phosphocreatine, and total creatine.

\section{Metabolic Spectrum According to PR Expression}

A positive $\mathrm{PR}$ was statistically associated with an elevated concentration of aspartate, serine, lysine, glucose, isoleucine, valine, alanine, proline, adenosine, arginine, creatine, and total creatine and a low concentration of taurine, acetate, threonine, NAA, NA-lysine, HB, myoinositol, ascorbate, scylloinositol, fumarate, $2 \mathrm{HG}$, choline, GPC, PC, total choline, and betaine.

We were then able to describe a metabolic profile associated with aggressive meningiomas (according to histological markers). Indeed, a high concentration of acetate, threonine, NA-lysine, HB, myoinositol, ascorbate, scylloinositol, and total choline and a low concentration of aspartate, serine, glucose, adenosine, arginine, alanine, and creatine were associated with a grade II/III meningioma with a Ki-67 rate $\geq 40 \%$ and a 


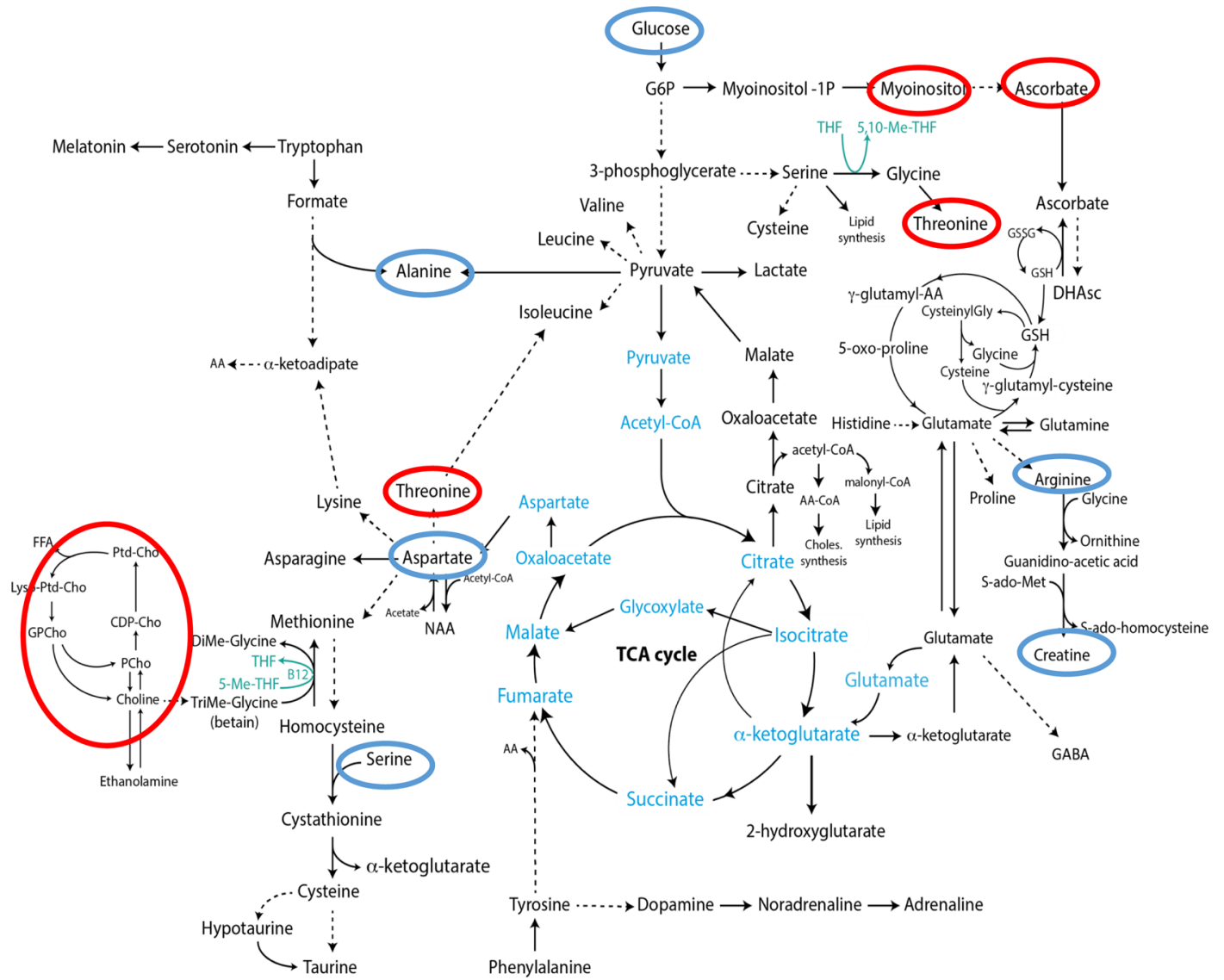

Figure 2. Metabolic pathways considering aggressive meningiomas (grade II/III, Ki-67 > 40\%, and negative PR expression).

Table 1. Management Strategy of Meningiomas Using High-Resolution Angle Spinning Spectrometry

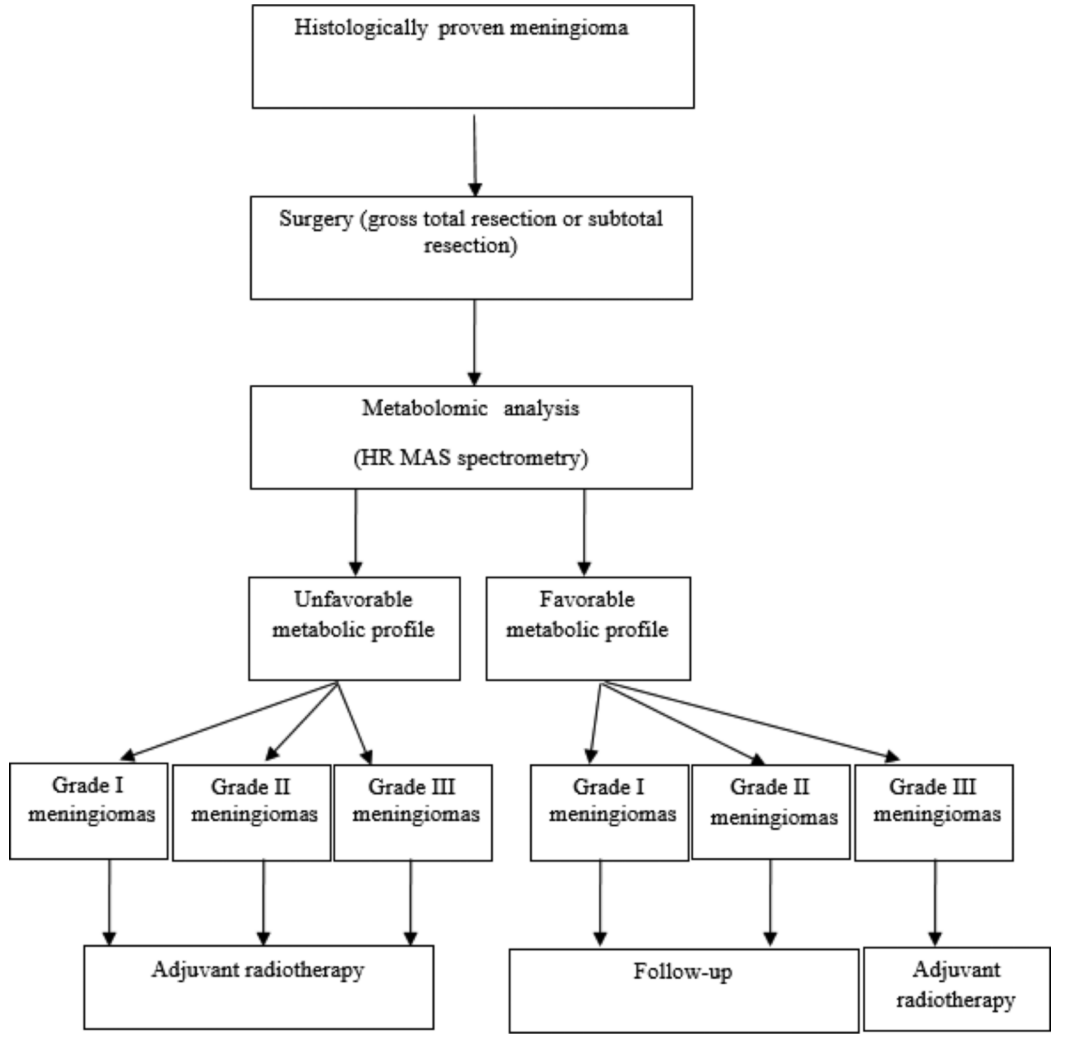



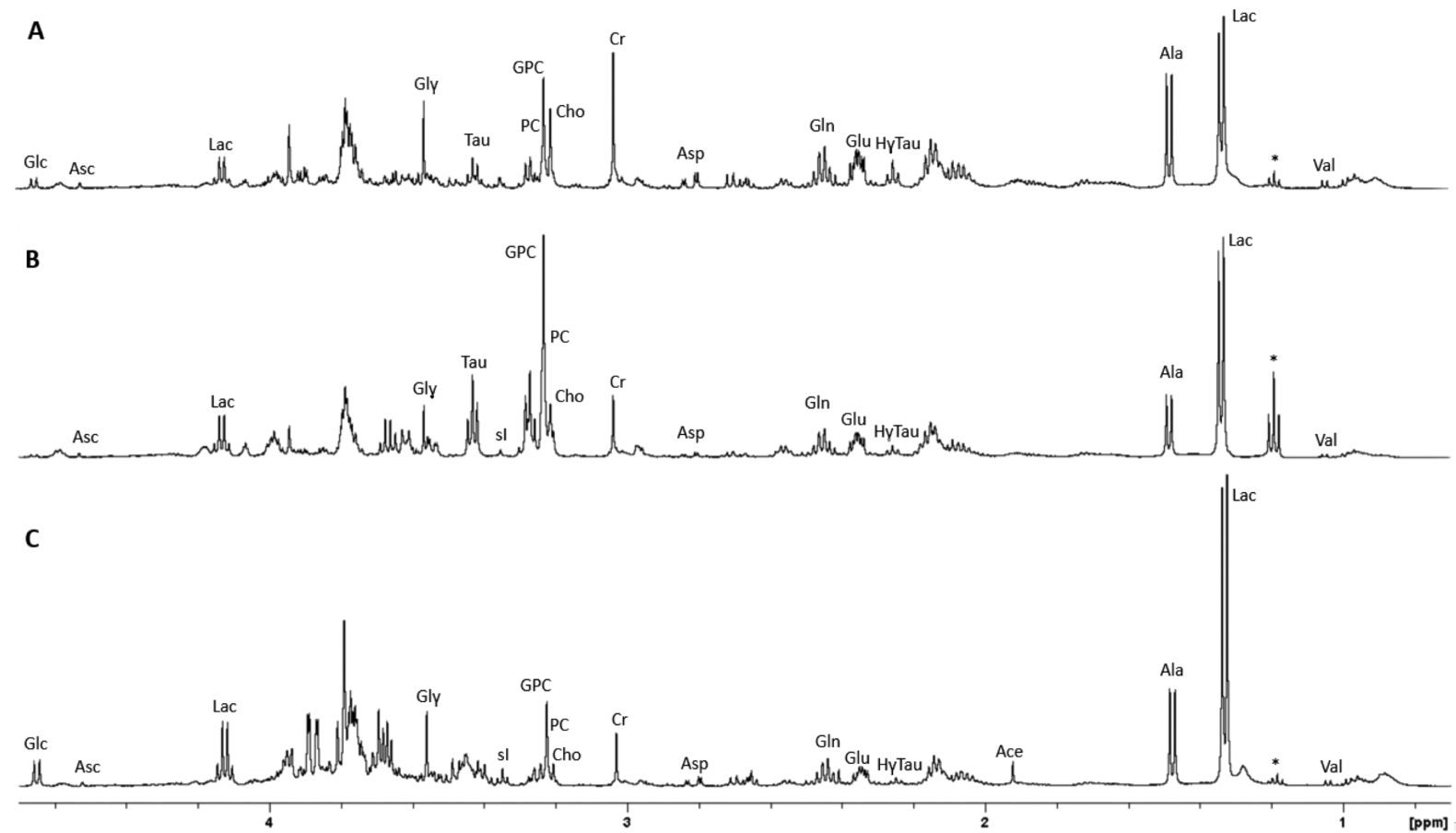

Figure 3. Three representative spectra of grade I meningiomas with low Ki-67 rates [(A) 4, (B) 2, and (C) $3 \%$, respectively) and positive PR expression.
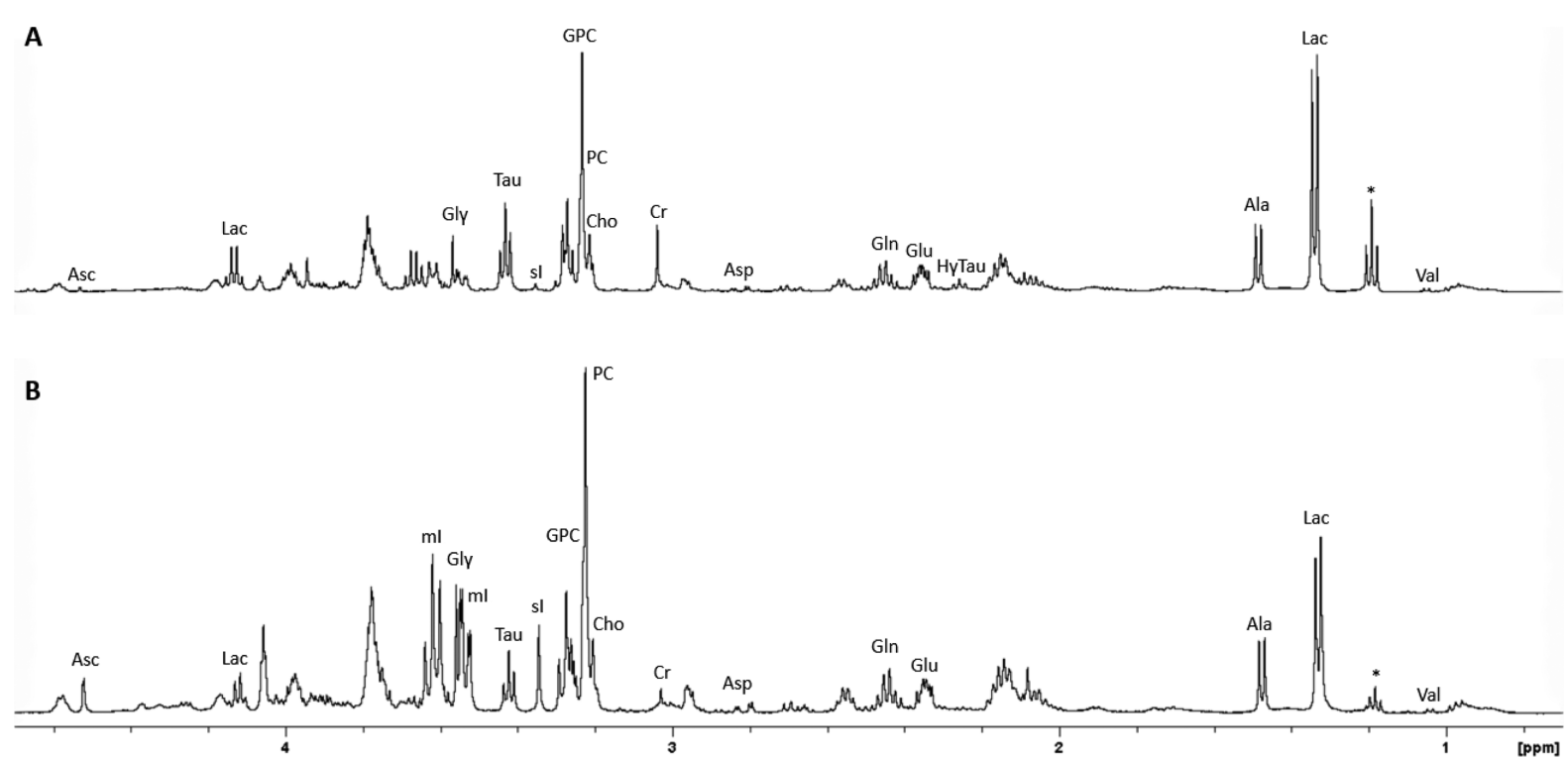

Figure 4. Comparative spectra of grade I meningiomas [(A) Ki-67 rate: 4\%; positive PR expression] and grade III meningiomas [(B) Ki-67 rate: $80 \%$; negative PR expression].

negative PR expression (Figure 1). Aside from threonine ( $p=$ 0.01 ), no metabolite is associated with OS. There was no significant metabolic pathway (Figure 2).

We proposed a management strategy by using metabolic analysis according to HRMAS NMR spectrometry (Table 1). Indeed, the metabolic profile could permit to distinguish between meningiomas which relapse and those which do not, in order to adapt the therapeutic strategy.

Moreover, we showed that all meningiomas with a low histological grade and a low Ki-67 proliferation index did not have a similar metabolomic profile (Figure 3). Indeed, some benign meningiomas had a comparative metabolomic spectrum compared to the high-grade meningiomas (Figure 4).

\section{DISCUSSION}

We retrospectively studied the metabolic profile for 62 meningiomas by using HRMAS NMR. In our study, we correlated ex vivo spectrometry data with three histological features (histological grade, Ki-67 rate, and PR expression). We defined an aggressive meningioma as a tumor with a high histological grade, a Ki-67 rate $\geq 40 \%$, and a negative PR expression. In our study, these three histological markers were associated with OS. According to the WHO 2007 classification (revised in 2016), a high-grade meningioma (II/III) is associated with a poor prognosis and a high risk of recurrence. $^{7,20,21}$ Two studies described Ki-67 proliferative 
index as a prognosis factor of recurrence. ${ }^{22,23}$ Moreover, in a retrospective study including 48 patients, Iplikcioglu et al. showed that a positive PR expression was statistically associated with low histological grade. ${ }^{24}$

In our study, a significantly elevated concentration of acetate, threonine, NA-lysine, HB, myoinositol, ascorbate, scylloinositol, and total choline and a low concentration of aspartate, glucose, isoleucine, valine, alanine, adenosine, and arginine were associated with meningiomas which had poor histological prognosis markers (a high histological grade, Ki-67 $\geq 40 \%$, and a negative PR expression). NA-lysine is produced by the acetylation of lysine. The impact of NA-lysine in tumor cell remains poorly understood for now. ${ }^{25}$ Myoinositol is a membrane component which participates in several cellular processes such as metabolic homeostasis, mRNA export, and stress response. ${ }^{26}$ This role in the carcinogenesis remains unknown. Ascorbate is an essential nutriment. This metabolite is an inhibitor factor of the hypoxia-inducible factor system, and it decreased VEGF expression. Ascorbate also had a cytotoxic activity because of an oxidative-related mechanism. ${ }^{27}$ The production of phosphorylcholine is an essential component in the induction of DNA synthesis and is therefore responsible of cell proliferation. ${ }^{28}$ An increased total choline expression reflects a tumoral hypoxia, and the GPC/PC ratio is related to the tumoral aggressiveness. These findings are consistent with our result. Aspartate decreased tumor proliferation, but the physiopathology remains unknown. ${ }^{29}$ This finding is also consistent with our results. Glucose and alanine produce pyruvate which is transformed into lactate in the cell without oxygen such as tumor cell. ${ }^{30}$ In our study, we observed that a low concentration of glucose and alanine was associated with an aggressive tumor.

There is actually a lack of data considering ex vivo spectroscopy for meningiomas. Monleón et al. studied 30 meningioma samples by using HRMAS NMR. The authors analyzed only 10 metabolites. Among the 30 meningiomas, 23 were classed grade I and 7 were classed grade II. The authors showed that the concentrations of PC (3.2 ppm) $(p=0.040)$ and phosphoethanolamine (4.01 ppm) $(p=0.016)$ were statistically higher for atypical meningiomas compared to benign meningiomas. These metabolites are implicated in the synthesis and degradation of phospholipids. Glutamine (2.44 ppm) $(p=0.045)$, glutamate $(2.35 \mathrm{ppm})(p=0.008)$, and GSH $(2.55 \mathrm{ppm})(p=0.019)$ were statistically present in a higher concentration for grade II meningiomas compared to grade I meningiomas. GSH is an antioxidant and plays a role in free radical protection. Moreover, the concentration of taurine $(3.42 \mathrm{ppm})(p=0.0041)$ was statistically higher for atypical meningiomas compared to benign meningiomas. ${ }^{14}$ Aside from $\mathrm{PC}$, the findings were contrast with our results. These differences could be explained by the fact that we included all grades of meningiomas (grades I to III). Moreover, our work study included twice as many tumor samples. Pfisterer et al. analyzed 68 meningioma samples (46 grade I, 14 grade II, and 8 grade III) by using proton magnetic resonance spectroscopy ( 8 metabolites). The mean concentrations of alanine and creatine were statistically higher in benign meningiomas compared to grade II and III meningiomas ( $p$ $=0.002)$. Furthermore, the authors noted that the mean concentrations of creatine and alanine were statically lower in tumors which rapidly recurred compared to those that did not $(p<0.001) .{ }^{15}$ To complete these results, Pfisterer et al. studied the metabolic profile by using proton magnetic resonance spectrometry for 30 benign meningiomas to distinguish between clinically aggressive benign meningiomas and those with a good prognosis. The authors analyzed six metabolites. The creatine concentration was statistically lower for benign meningiomas which rapidly recurred $(p<0.05)$. Alanine tended to be lower for grade I tumors which recurred ( $p=$ $0.05)$. No metabolite was statistically associated with Ki-67 expression or brain invasion. ${ }^{31} \mathrm{We}$ described a similar result in our study; indeed, an elevated concentration of alanine was also associated with benign meningiomas. Moreover, we described a correlation between a high concentration of alanine and a low Ki-67 expression. However, in our study, creatine was not associated with the tumor grade, and a high concentration of this metabolite was statistically associated with a high Ki-67 expression.

Moreover, in our study, we described three different spectra representative of a meningioma with nonaggressive histological features (grade I, a low Ki-67 expression, and a positive PR expression). The concentration of the metabolites was different for these tumors, mainly total choline, glucose, and creatine. One grade I meningioma with a low Ki-67 expression had no expression of glucose; this suggests an aggressive tumor behavior. Moreover, one grade I meningioma had a low concentration of creatine, which suggests tumor hypoxia and aggressive behavior. Despite different metabolite concentrations, all grade I meningiomas had a very low concentration of myoinositol and scylloinositol and a positive glycerophosphocholine/PC (GPC/PC) ratio.

Furthermore, we described a similar metabolic spectra between a grade I meningioma ( $\mathrm{Ki}-67$ rate $4 \%$ and a positive PR expression) and a grade III meningioma (Ki-67 rate $80 \%$ and a negative PR expression). We obtained similar glutamate, glutamine, and total choline concentrations between both tumors. However, the GPC/PC ratio was different. Indeed, the ratio was positive for grade I meningiomas and negative for grade III meningiomas. A negative GPC/PC ratio is associated with malignant tumors. Furthermore, the creatine concentration was also lower for grade III meningiomas compared to grade I meningiomas. This suggests that hypoxia is higher for grade III meningiomas. Glycine, myoinositol, scylloinositol, and ascorbate concentrations were also higher for grade III meningiomas compared to grade I meningiomas. Taurine and hypotaurine concentrations were lower for grade III meningiomas in comparison with grade I meningiomas.

Finally, we proposed a metabolic profile associated with poor histological prognosis markers. This signature could be used to discuss a personalized therapeutic management. However, future studies are needed to confirm these results in order to include spectrometry and histological analysis to improve the classification of meningiomas.

\section{CONCLUSIONS}

Meningiomas are the most common benign brain tumors. However, some tumors can have a clinically aggressive behavior. Histological markers such as Ki-67, PR expression, or tumor grade are correlated with prognosis. We described a metabolic profile associated with poor histological prognosis markers for meningioma. Metabolic analysis could be useful to distinguish between clinically aggressive meningiomas and those which did not recur in order to adapt the initial treatment. Future studies are needed to determine an association between this metabolic signature and survival data. 


\section{AUTHOR INFORMATION}

\section{Corresponding Author}

*E-mail: laura_2708@hotmail.fr. Phone: +33 (0)3 881276 65. Fax: +33 (0)388 128954 .

ORCID

Laura Bender: 0000-0003-3486-8505

Notes

The authors declare no competing financial interest.

\section{ACKNOWLEDGMENTS}

The authors of the manuscript have no financial interest or arrangement with a company whose product was used in a study or is referred to in an article, any financial interest in or arrangement with a competing company, any other financial connections, direct or indirect, or other situations that might raise the question of bias in the work reported or the conclusions, implications or opinions stated including pertinent commercial, governmental, private or other sources of funding for the individual author(s) or for the affiliated department(s) or organization(s), personal relationships, or direct academic competition.

\section{REFERENCES}

(1) Dolecek, T. A.; Propp, J. M.; Stroup, N. E.; Kruchko, C. CBTRUS Statistical Report: Primary Brain and Central Nervous System Tumors Diagnosed in the United States in 2005-2009. Neuro Oncol. 2012, 14, v1-v49.

(2) Wiemels, J.; Wrensch, M.; Claus, E. B. Epidemiology and Etiology of Meningioma. J. Neuro Oncol. 2010, 99, 307-314.

(3) Kshettry, V. R.; Ostrom, Q. T.; Kruchko, C.; Al-Mefty, O.; Barnett, G. H.; Barnholtz-Sloan, J. S. Descriptive Epidemiology of World Health Organization Grades II and III Intracranial Meningiomas in the United States. Neuro Oncol. 2015, 17, 11661173.

(4) Holden, J.; Dolman, C. L.; Churg, A. Immunohistochemistry of Meningiomas Including the Angioblastic Type. J. Neuropathol. Exp. Neurol. 1987, 46, 50-56.

(5) Maxwell, M.; Galanopoulos, T.; Neville-Golden, J.; Antoniades, H. N. Expression of Androgen and Progesterone Receptors in Primary Human Meningiomas. J. Neurosurg. 1993, 78, 456-462.

(6) Ragel, B. T.; Jensen, R. L. Aberrant Signaling Pathways in Meningiomas, Ragel and al. Journal of Neuro-Oncology. J. Neuro Oncol. 2010, 99, 315-324.

(7) Bi, W. L.; Zhang, M.; Wu, W. W.; Mei, Y.; Dunn, I. F. Meningioma Genomics: Diagnostic, Prognostic, and Therapeutic Applications. Front. Surg. 2016, 3, 40.

(8) Backer-Grøndahl, T.; Moen, B. H.; Torp, S. H. The Histopathological Spectrum of Human Meningiomas. Int. J. Clin. Exp. Pathol. 2012, 5, 231-242.

(9) Dietz, C.; Ehret, F.; Palmas, F.; Vandergrift, L. A.; Jiang, Y.; Schmitt, V.; Dufner, V.; Habbel, P.; Nowak, J.; Cheng, L. L. Applications of High-Resolution Magic Angle Spinning MRS in Biomedical Studies II-Human Diseases. NMR Biomed. 2017, 30, e3784.

(10) Giskeødegård, G. F.; Cao, M. D.; Bathen, T. F. HighResolution Magic-Angle-Spinning NMR Spectroscopy of Intact Tissue. In Metabonomics; Bjerrum, J. T., Ed.; Springer New York: New York, NY, 2015; Vol. 1277, pp 37-50.

(11) Chen, W.; Lu, S.; Wang, G.; Chen, F.; Bai, C. Staging Research of Human Lung Cancer Tissues by High-Resolution Magic Angle Spinning Proton Nuclear Magnetic Resonance Spectroscopy (HRMAS $1 \mathrm{H} \mathrm{NMR)} \mathrm{and} \mathrm{Multivariate} \mathrm{Data} \mathrm{Analysis.} \mathrm{Asia} \mathrm{Pac.} \mathrm{J.}$ Clin. Oncol. 2017, 13, e232-e238.

(12) Chae, E. Y.; Shin, H. J.; Kim, S.; Baek, H.-M.; Yoon, D.; Kim, S.; Shim, Y. E.; Kim, H. H.; Cha, J. H.; Choi, W. J.; et al. The Role of
High-Resolution Magic Angle Spinning 1H Nuclear Magnetic Resonance Spectroscopy for Predicting the Invasive Component in Patients with Ductal Carcinoma In Situ Diagnosed on Preoperative Biopsy. PLoS One 2016, 11, e0161038.

(13) Lee, C. W.; Lee, J. S.; Woo, C. W.; Kim, S. High-Resolution Magic Angle Spinning Nuclear Magnetic Resonance Spectroscopy for the Metabolic Assessment of Acute Rejection After Cardiac Transplantation in Rats. Transplant. Proc. 2017, 49, 1935-1941.

(14) Monleón, D.; Morales, J. M.; Gonzalez-Darder, J.; Talamantes, F.; Cortés, O.; Gil-Benso, R.; López-Ginés, C.; Cerdá-Nicolás, M.; Celda, B. Benign and Atypical Meningioma Metabolic Signatures by High-Resolution Magic-Angle Spinning Molecular Profiling. J. Proteome Res. 2008, 7, 2882-2888.

(15) Pfisterer, W. K.; Nieman, R. A.; Scheck, A. C.; Coons, S. W.; Spetzler, R. F.; Preul, M. C. Using Ex Vivo Proton Magnetic Resonance Spectroscopy to Reveal Associations between Biochemical and Biological Features of Meningiomas. Neurosurg. Focus 2010, 28, E12.

(16) Ruhland, E.; Bund, C.; Outilaft, H.; Piotto, M.; Namer, I.-J. A Metabolic Database for Biomedical Studies of Biopsy Specimens by High-Resolution Magic Angle Spinning Nuclear MR: A Qualitative and Quantitative Tool. Magn. Reson. Med. 2019, 82, 62.

(17) Cicek, A. E.; Bederman, I.; Henderson, L.; Drumm, M. L.; Ozsoyoglu, G. ADEMA: An Algorithm to Determine Expected Metabolite Level Alterations Using Mutual Information. PLoS Comput. Biol. 2013, 9, e1002859.

(18) Kanehisa, M.; Goto, S. KEGG: Kyoto Encyclopedia of Genes and Genomes. Nucleic Acids Res. 2000, 28, 27-30.

(19) Kanehisa, M.; Goto, S.; Sato, Y.; Kawashima, M.; Furumichi, M.; Tanabe, M. Data, Information, Knowledge and Principle: Back to Metabolism in KEGG. Nucleic Acids Res. 2014, 42, D199-D205.

(20) Louis, D. N.; Ohgaki, H.; Wiestler, O. D.; Cavenee, W. K.; Burger, P. C.; Jouvet, A.; Scheithauer, B. W.; Kleihues, P. The 2007 WHO Classification of Tumours of the Central Nervous System. Acta Neuropathol. 2007, 114, 97-109.

(21) Louis, D. N.; Perry, A.; Reifenberger, G.; von Deimling, A.; Figarella-Branger, D.; Cavenee, W. K.; Ohgaki, H.; Wiestler, O. D.; Kleihues, P.; Ellison, D. W. The 2016 World Health Organization Classification of Tumors of the Central Nervous System: A Summary. Acta Neuropathol. 2016, 131, 803-820.

(22) Terzi, A.; Saglam, E. A.; Barak, A.; Soylemezoglu, F. The Significance of Immunohistochemical Expression of Ki-67, P53, P21, and P16 in Meningiomas Tissue Arrays. Pathol., Res. Pract. 2008, 204, $305-314$.

(23) Wang, Y.-C.; Chuang, C.-C.; Wei, K.-C.; Hsu, Y.-H.; Hsu, P.W.; Lee, S.-T.; Wu, C.-T.; Tseng, C.-K.; Wang, C.-C.; Chen, Y.-L.; et al. Skull Base Atypical Meningioma: Long Term Surgical Outcome and Prognostic Factors. Clin. Neurol. Neurosurg. 2015, 128, 112-116.

(24) Iplikcioglu, A. C.; Hatiboglu, M. A.; Ozek, E.; Ozcan, D. Is Progesteron Receptor Status Really a Prognostic Factor for Intracranial Meningiomas? Clin. Neurol. Neurosurg. 2014, 124, 119-122.

(25) Gil, J.; Ramírez-Torres, A.; Encarnación-Guevara, S. Lysine Acetylation and Cancer: A Proteomics Perspective. J. Proteom. 2017, 150, 297-309.

(26) Bizzarri, M.; Fuso, A.; Dinicola, S.; Cucina, A.; Bevilacqua, A. Pharmacodynamics and Pharmacokinetics of Inositol(s) in Health and Disease. Expert Opin. Drug Metabol. Toxicol. 2016, 12, 11811196.

(27) Mastrangelo, D.; Pelosi, E.; Castelli, G.; Lo-Coco, F.; Testa, U. Mechanisms of Anti-Cancer Effects of Ascorbate: Cytotoxic Activity and Epigenetic Modulation. Blood Cells Mol. Dis. 2018, 69, 57-64.

(28) Cuadrado, A.; Carnero, A.; Dolfi, F.; Jiménez, B.; Lacal, J. C. Phosphorylcholine: A Novel Second Messenger Essential for Mitogenic Activity of Growth Factors. Oncogene 1993, 8, 2959-2968.

(29) Sullivan, L. B.; Luengo, A.; Danai, L. V.; Bush, L. N.; Diehl, F. F.; Hosios, A. M.; Lau, A. N.; Elmiligy, S.; Malstrom, S.; Lewis, C. A.; et al. Aspartate Is an Endogenous Metabolic Limitation for Tumour Growth. Nat. Cell Biol. 2018, 20, 782-788. 
(30) Adeva-Andany, M.; López-Ojén, M.; Funcasta-Calderón, R.; Ameneiros-Rodríguez, E.; Donapetry-García, C.; Vila-Altesor, M.; Rodríguez-Seijas, J. Comprehensive Review on Lactate Metabolism in Human Health. Mitochondrion 2014, 17, 76-100.

(31) Pfisterer, W. K.; Hendricks, W. P.; Scheck, A. C.; Nieman, R. A.; Birkner, T. H.; Krampla, W. W.; Preul, M. C. FLUORESCENT IN SITU HYBRIDIZATION AND EX VIVO 1H MAGNETIC RESONANCE SPECTROSCOPIC EXAMINATIONS OF MENINGIOMA TUMOR TISSUE. Neurosurgery 2007, 61, 1048-1061. 